Copyright ACM, 2014. This is the author's version of the work. It is posted here by permission of ACM for your personal use. Not for redistribution. The definitive version was published in Proceedings of the 26th Australian Computer-Human Interaction Conference on Designing Futures: the Future of Design, ISBN: 978-1-4503-0653-9, dx.doi.org/10.1145/2686612.2686695.

\title{
Experiences Using Emerging Technology
}

\author{
Jake Araullo \\ Griffith University \\ Kessels Rd, Nathan, 4111 \\ Jake.Araullo@griffithuni.edu.au
}

\author{
Leigh Ellen Potter \\ Griffith University \\ Kessels Rd, Nathan, 4111 \\ L.Potter@griffith.edu.au
}

\begin{abstract}
Emerging technologies are being developed at a rapid pace, driven by strong innovation in the hardware sector, resulting in an increase in the range of input devices that people can use to interact with computers and applications. Unfortunately, there are no established interaction standards or best practices when developing software for emerging technologies, and user experience suffers as a result. This paper presents the findings from an early case study exploring the experiences of a group of individuals when playing games using emerging technology: namely the Oculus Rift and the Leap Motion controller. This case study provides insight into the usability of the interaction between game and device.
\end{abstract}

\section{Author Keywords}

Emerging Technology; Natural User Interface

\section{ACM Classification Keywords}

H5.2 User interfaces

\section{INTRODUCTION}

This paper presents the experiences of a group of individuals when using emerging technology devices. As the technology industry has innovated and grown, particularly in the past decade, a number of new hardware input peripherals have come to market that allow more immersive, interactive and natural computing experiences than ever before. Hardware input devices such as Microsoft Kinect, Oculus Rift and Leap Motion among others have spearheaded the drive of 'natural' input devices into the consumer market over the last few years. They represent a new pinnacle in hardware innovation and state-of-the-art sensory and input technology (O'Hara, Harper, Mentis, Sellen and Taylor, 2013).

This growth and innovation in input devices has seen an increase in the level of inconsistency between interfaces and input systems, even between similar devices. Often user interaction becomes fragmented and user expectations of a system do not match reality (Wigdor and Morrison, 2010). Currently, many applications using emerging technologies make use of traditional interface metaphors, iconography and elements. Using these elements relies on the prior-knowledge of users and exploits their expectations and familiarities (Hofmeester and Wixon, 2010). The software that accompanies the new devices often lacks cohesion, standard best practices and detailed usability design. Interfaces and interaction techniques vary between apps and devices, and ultimately user experience suffers (Metz, 2013).

Usability when designing interfaces for emerging technologies in particular is an area that has not been extensively studied to date. The research presented in this paper is the first stage of a larger project that is currently underway to begin working towards establishing consensus standards when using emerging technologies, and to establish a set of characteristics that an effective natural user interface for emerging technologies should exhibit in order to facilitate the user experience. This paper presents the usability issues that were encountered when interacting with two specific emerging technology devices from the point of view of a group of individuals. This forms the basis of further work towards identifying key characteristics.

Definitions of emerging technology and natural user interfaces will be given, prior to an overview of the research study. Observations of participant interactions with two specific emerging technologies, the Oculus Rift and Leap Motion controller, will be described and the issues experienced by the study participants will be presented. The next stage of the project involves the development of a set of recommendations for natural user interface development based on these observations, and future work will see the development of interaction prototypes to explore the suitability of the recommendations.

\section{EMERGING TECHNOLOGY}

Emerging technology has been defined as "radical technologies that emerge from new technologies or incremental technologies that arise from the convergence of existing technologies" (Srinivasan, 2008). The defining feature of such technology is "the potential to create a new industry or transform an existing one" (Day and Schoemaker, 2000). The nature of the technology may be dependent on its context and application (Halaweh, 2013), with a technology considered emerging in certain geographic markets and simultaneously mature in a market where application and innovation of the technology is more pervasive (Srinivasan, 2008).

Significant research has been completed to find "faster, more natural, and more convenient means for a user to transmit information to a computer" (Jacob, 1996). Input and control hardware devices act as an intermediary, 
turning human interaction into software commands. The primary task of a computer input or control device "is to move information from the brain of the user to the computer" (Jacob, 1996), traditionally using a computer mouse or keyboard, a television remote or a joystick. Emerging technologies are introducing a new range of devices, with their own unique interactions. We can define emerging input hardware to be: a device with potential to instigate change in the fundamental methods with which we interact with our computer systems.

\section{NATURAL USER INTERFACES}

It is important when exploring emerging technology to consider both the new hardware and the software that may use this hardware. Natural User Interfaces (NUIs) are software systems that facilitate interaction between hardware and the digital world (Wigdor and Wixon, 2011). What uniquely identifies a natural user interface as opposed to a standard user interface is that the NUI "[takes] advantage of modern input technology" (Wigdor and Wixon, 2011) to provide an experience that integrates technology into our lives in a familiar and comfortable way. In order to explore the experience of using an emerging technology, we must also consider the experience of the natural user interface.

Emerging hardware is often partnered and developed alongside an accompanying NUI in order to leverage new and unconventional hardware applications. Current approaches to NUI development often attempt to capitalise on new modalities presented by emerging hardware in NUI design to provide a natural experience (Jain, Lund and Wixon, 2011).

\section{THE CASE STUDY}

This paper presents findings gathered as part of a larger study. In order to "seek out and interpret the meanings that people bring to their own actions" (Payne and Payne, 2004) a qualitative case study approach has been applied. This allows examination of phenomenon within the context in which it occurs (Yin, 2009). This study observed participant interactions with several virtual reality games for usability issues, before identifying and correlating similar issues across participants. Participants completed a questionnaire after the sessions to gather their own opinions of the interaction and the session.

Sessions were conducted in participant/researcher pairs. Participants were seated in front of a laptop computer at a desk. Attached to the laptop computer were a Leap Motion Controller device and an Oculus Rift Virtual Reality headset device. The Oculus Rift Development Kit 1 was used in this study. Participants were asked to complete four tasks in four different commercially available video game applications: Minecraft, and Aaaaaaculus! using the Oculus Rift, and Volantes, and Out of the Blocks using the Leap Motion controller. These were selected in order to maximise participant exposure to current industry standard NUIs paired with emerging hardware. In a session each participant would complete the following tasks:

- Minecraft: Start a new game. Walk forwards, backwards, left and right in game;
- Aaaaaculus!: Start a new game. Score 1000 points;

- Volantes: Start a new game. Move your ship to a goal area. Fire your weapon;

- Out of the Blocks: Start a new game. Walk forwards, backwards, left and right in game. Fire your weapon.

The tasks took approximately 25 minutes to complete, with this timeframe consistent regardless of the participants prior gaming experience.

In total 16 participants took part in the case study (3 female and 13 male), identified through pseudonyms. Participants ranged in age from $20-34$ years old, with the majority between 20 and 25 . Half the participants were university students and half were employed, with occupations in retail, accounting, medical science, and manual labour. Participants were required to have a level of computer familiarity consistent with regular usage (at least once per week). Participants without previous exposure to the Leap Motion Controller or Oculus Rift virtual reality headset were given a brief introduction to the technology, including an outline of correct use procedures for both devices.

During sessions participant interactions were observed and notes were taken of their experience using observation forms. A questionnaire was provided for each game interaction. The questionnaire allowed users to document their individual experience and perceptions of the interaction.

\section{THE PARTICIPANT EXPERIENCE}

Through participant behaviour and comments during the case study and feedback through the questionnaire, a number of usability issues were noted.

\section{Instructions and Ambiguous Language}

Participants demonstrated a higher level of game competency, and described a consistently better experience using applications that provided a clear form of instruction. In some cases they had difficulty interpreting potentially unhelpful instructions, and these were perceived as more of a hindrance. When clear instructions were provided participants were generally able to use a fuller set of system functionality. The absence of instructions was noticed and often commented upon by participants.

Throughout testing, participants responded positively to the inclusion of diagrams accompanying text instructions. Participants that identified as being unfamiliar with the technology were particularly vocal in their support for diagrams. One participant in particular, Jack, struggled to perform the 'shooting' game mechanic in the Leap Motion application Volantes, despite the presence of text instructions. Later in the testing session Jack was asked to perform a very similar shooting mechanic in the Leap Motion game Out of the Blocks. The primary difference in the presentation of these actions was the inclusion of an animated illustration depicting a hand moving in the required motion above a Leap Motion controller. Jack was able to immediately mimic the action and engage the system to perform as required. 
Both Leap Motion games used in testing provided instructions regarding game purpose and operation, while both Oculus Rift games provided no player instruction. Participants did not often see a benefit in the explicit Leap Motion instructions due to ambiguity in language. An example of this occurred for many participants using Volantes. The game instructed participants to "Push hands forward to shoot". Participants struggled to understand how their hand should sit during this action as well as the speed of the action. Jack couldn't get the game to shoot: "What does push hands forward actually mean? What is forward?" Participants found the language of Volantes to be frustratingly non-specific and assumptive regarding their familiarity with the hardware platform, and found the instructions of Out of the Blocks cryptic and unclear in relation to the goal they wanted to achieve.

\section{Simplicity and Complexity}

Participants stated that Aaaaaaculus! had the simplest control system and concept of the four applications, and described a high level of satisfaction in their experience with this game. Participants were vocal about this game being "simple (Kate) and "easy to understand" (Chris). This perceived simplicity of concepts, control systems and interaction mechanics enhanced their overall perceptions of the game.

Complexity in this context involves the deliberate reduction of system elements that are superfluous to core user experience. Participants found excessive interface elements (buttons and labels) as well as elaborate input schemes all increased perceived complexity. Simplicity resulted in higher levels of satisfaction, and higher perceived complexity led to participants underutilising portions of the system or ignoring instructions. The many mechanics of a system found both Jack and Kate ignoring on screen instructions and failing to retain information, resulting in a frustrating, confused experience.

The two systems described as least complex were Minecraft and Volantes and participants commented that they were more comfortable achieving their goals using these systems. Participants described Aaaaaaculus! and Out of the Blocks as more complex, and expressed a level of discomfort using these systems. During testing Michael found interacting with Out of the Blocks' control system frustratingly complex and commented that operating the control system as well as following on screen instructions was "too much distraction" and resulted in him forgetting the goal of the game.

\section{Multiple Input Peripherals}

All of the applications used in these sessions used multiple input peripherals and existing traditional computing metaphors and interaction patterns. Minecraft relied heavily on keyboard, mouse and head tracking inputs. Sarah, Ben and Justin all leaned heavily on keyboard and mouse input, barely using the head-tracking technology (the defining feature of the Oculus Rift). In Minecraft, mouse and head tracking performed the same function. This doubling up of controls and resulting double impact of actions resulted in nausea, hindered participant comfort, and affected the extent of system functionality that a participant would use.
Multiple input peripherals led to participant confusion during the sessions. While wearing the Oculus Rift participants were unable to see the keyboard, which was necessary for in-game controls. Participants were quite vocal regarding their struggles to not only locate the keyboard, but use particular keys accurately.

Out of the Blocks presented users with an oversize cursor at the main menu, a metaphor traditionally associated with mouse use, but expected users to interact gesturally with their hands. This incongruent set of expectations not only left users confused initially but also set the wrong expectations moving forward, with participants attempting to make a mouse click gesture in air for selection. While gestural input was clearly dominant in Out of the Blocks, participant reaction to inclusion of traditional metaphors such as a cursor clearly had a negative impact on their use of the system.

\section{Assumed Familiarity and Expectations}

All of the applications tested experienced at least some issues around assumed familiarity with the hardware system or the method of interaction with the application. Volantes required users to engage the application, using the Leap Motion, with two hands, while Out of the Blocks requires users to use only a single hand. Most participants used the wrong number of hands when interacting with a system. The only application to explicitly identify interaction expectations was Volantes, telling users to hold two hands above the device.

There was no standardised interaction framework among the applications tested, and as a result, participants often struggled and became audibly frustrated transitioning between applications as their expectations from one application were not met in the next. When using the Oculus Rift, Sarah found that in Minecraft she was able to point her head and press the forward key to move in that direction. She commented that she liked this control system as it was "easy to use". When she moved on to Aaaaaculus! she found head movements had no effect on the direction she moved. She expressed frustration that the game was not reacting as she anticipated, describing Aaaaaaculus! as 'very complex' and 'harder to use'.

Participants who often played video games reacted differently to those who did not play video games frequently. Despite unfamiliarity with particular emerging technologies, those with notable video game experience were able to grasp new modes of interaction more quickly. They also appeared to overlook many usability issues they encountered during game play if they found a game fun or exciting. Often participants who had less experience using emerging technologies (such as Jack) were unable to identify why the issues they were experiencing were even occurring.

\section{Interface Modification}

One current approach to NUI implementation is porting existing applications with little modification, meaning developers are yet to "make good use of a completely new form of interaction" (Metz, 2014). This was a clear issue throughout the case study; if application interfaces were not reimagined to suit their partnered emerging 
technology, or if adjunct interface artefacts remained in use, numerous facets of user experience were affected, particularly a participant's ability to use the system.

Minecraft is a game ported to work with the Oculus Rift, and a clear majority of participants expressed frustration with user interface elements that were not optimised for the new technology. The application loading screen made many participants feel disoriented as it was not stereoscopic unlike the rest of the application. Many participants struggled to read the menu text, largely unchanged during porting from the traditional desktop version of the game. Participants reported the text to be blurry and the buttons to be too numerous. Participants often resorted to guessing which UI elements would accomplish the action they were attempting. In contrast, the interface of Aaaaaaculus! was completely redesigned for the Oculus Rift. Participants reported no difficulties reading or using this optimised system.

\section{Clear Goal or Objective}

A factor that often impacted participant satisfaction was a lack of clear goals or objectives. Participants commented that they had no way of "knowing whether what I was doing was correct" (Sarah) and they sometimes "got lost and confused" (Kate). In Out of the Blocks and Aaaaaaculus! a goal was not clearly identified and participants found vague or nonspecific instructions unhelpful and overwhelmingly frustrating.

\section{Physical Considerations}

As input devices reside in the physical world, it is important to consider the physicality of devices when designing interfaces for them. Volantes required users to have two hands raised over the Leap Motion controller at all times, or the system would not function. Just under half of participants found this uncomfortable after even short periods of time. Out of the Blocks eased this particular concern by requiring only a single hand for use, and allowing participants to swap hands mid-game. However, some participants found that Out of the Blocks's system of movement based on hand displacement from an in-air centre-point was more "demanding" as it required more effort to hold their hand very still at an arbitrary point in-air.

Minecraft required the use of multiple input peripherals. Participants were able to both turn their head and use the mouse to rotate and 'look' in game, however, a combination of both often left players disoriented or nauseated. Aaaaaaculus! left the necks of some participants sore, due to constantly looking directly down while falling in-game.

\section{Conclusion}

From observing participant interactions with emerging technology devices, a range of issues have been identified that should be addressed when developing NUIs for these technologies. The presence of clear instructions and diagrams assisted participants in interacting fully with the game and the device. Conversely, ambiguous language left participants as or more frustrated than they were without any instruction at all, and this can hamper full, unimpeded interaction with the NUI of the system.
Greater perceived system complexity hampered use of full system functionality and left participants feeling frustrated and less confident achieving their goals. Participant responses and actions during sessions also indicated that application use was negatively affected by the inclusion of mixed traditional and non-traditional input peripherals, and by reliance on traditional interface metaphors when using emerging technologies. A lack of standardisation between applications led to significant participant frustration and impeded system use. User interface elements must be carefully considered and optimised for their paired emerging technology system.

The presence of clearly defined objectives was a recurring theme among participants, affecting their lasting impression of a system. In order to build user confidence and ultimately satisfaction, it is imperative that goals, objectives and paths through an application are clearly identified. Applications must also cater for physical considerations such as fatigue and nausea, as participant experience and comfort can be negatively affected. When designing interfaces for emerging hardware technologies it is imperative to consider user comfort to maximise usability and positive user experience.

\section{REFERENCES}

Day, G. S. and Schoemaker, P. J. H. A Different Game. Wiley, (2000).

Halaweh, M. Emerging Technology: What is it? Journal of Technology Management and Innovation, 8, 3 (2013), 108-115.

Hofmeester, K. and Wixon, D. Using Metaphors to Create a Natural User Interface for Microsoft Surface. In Proc CHI 2010, ACM (2010).

Jacob, R. J. K. The Future of Input Devices. ACM Computing Surveys, 28, 4 (1996), 4.

Jacob, R. J. K. Human-Computer Interaction: Input Devices. ACM Computing Surveys, 28, 1 (1996), 177179.

Jain, J., Lund, A. and Wixon, D. The Future of Natural User Interfaces. In Proc CHI 2011, ACM (2011).

Metz, R. Look before you Leap Motion. MIT, (2013).

Metz, R. For 3-D Interfaces, a long road to acceptance. MIT, (2014).

O'Hara, K., Harper, R., Mentis, H., Sellen, A. and Taylor, A. On the Naturalness of Touchless: Putting the Interaction Back into NUI. ACM Transactions on Computer-Human Interaction, 20, 1 (2013), 25.

Payne, G. and Payne, J. Key Concepts in Social Research. Sage, London, (2004).

Srinivasan, R. Sources, characteristics and effects of emerging technologies: Research opportunities in innovation. Industrial Marketing Management, 37, 6 (2008), 633-640.

Wigdor, D. and Morrison, G. Designing User Interfaces for Multi-touch and Surface-gesture Devices. ACM, (2010).

Wigdor, D. and Wixon, D. Brave NUI world: designing natural user interfaces for touch and gesture. Morgan Kaufmann, San Francisco, (2011).

Yin, R. K. Case Study Research. Sage, London, (2009). 